\title{
Antibacterial resistance worldwide: causes, challenges and responses
}

\author{
Stuart B Levy \& Bonnie Marshall
}

\begin{abstract}
The optimism of the early period of antimicrobial discovery has been tempered by the emergence of bacterial strains with resistance to these therapeutics. Today, clinically important bacteria are characterized not only by single drug resistance but also by multiple antibiotic resistance - the legacy of past decades of antimicrobial use and misuse. Drug resistance presents an everincreasing global public health threat that involves all major microbial pathogens and antimicrobial drugs.
\end{abstract}

Antimicrobial resistance is not new, but the number of resistant organisms, the geographic locations affected by drug resistance, and the breadth of resistance in single organisms are unprecedented and mounting ${ }^{1}$. Diseases and disease agents that were once thought to be controlled by antibiotics are returning in new leagues resistant to these therapies. In this review, we focus on the underlying principles and ecological factors that affect drug resistance in bacteria. It should be stressed, however, that antimicrobial resistance is also evident in other microorganisms - namely, parasites, fungi and viruses ${ }^{2}$.

Drug-resistant strains initially appeared in hospitals, where most antibiotics were being used ${ }^{3}$. Sulfonamide-resistant Streptoccoccus pyogenes emerged in military hospitals in the $1930 \mathrm{~s}^{4}$. Penicillinresistant Staphylococcus aureus confronted London civilian hospitals very soon after the introduction of penicillin in the $1940 \mathrm{~s}^{5}$. Similarly, Mycobacterium tuberculosis with resistance to streptomycin emerged in the community soon after the discovery of this antibiotic ${ }^{6}$.

Resistance to multiple drugs was first detected among enteric bacteria-namely, Escherichia coli, Shigella and Salmonella-in the late 1950 s to early $1960 \mathrm{~s}^{7-9}$. Such strains posed severe clinical problems and cost lives, particularly in developing countries. Nevertheless, the resistance problem was perceived by some, most notably those in the industrialized world, as a curiosity of little health concern confined to gastrointestinal organisms in distant countries. This attitude changed in the 1970s when Haemophilus influenzae and Neisseria gonorrhoeae, organisms that cause respiratory and genitourinary disease, respectively, emerged with resistance to ampicillin ${ }^{10,11}$ and, in the case of Haemophilus, with resistance to chloramphenicol and tetracycline as well $^{12,13}$. Fueled by increasing antimicrobial use, the frequency of resistance escalated in many different bacteria, especially in developing countries where antimicrobials were readily available without prescription. Poor sanitation conditions aided spread and small healthcare budgets prevented access to new effective but more expensive

Center for Adaptation Genetics and Drug Resistance, Departments of Molecular Biology and Microbiology and of Medicine, Tufts University School of Medicine, Boston, Massachusetts 02111, USA. Correspondence should be addressed to S.B.L. (stuart.levy@tufts.edu) or B.M. (bonnie.marshall@tufts.edu).

Published online 30 November 2004; doi:10.1038/nm1145 antibiotics $^{1}$. Since the 1980s, a re-emergence of tuberculosis has occurred that is often multidrug resistant (MDR) and enhanced by human immunodeficiency virus infection ${ }^{14}$. The severity of and difficulty in treating MDR strains necessitates the use of several, sometimes six to seven different, drugs ${ }^{15}$.

\section{Key problems of resistance in hospitals and communities}

Multiply resistant organisms render therapy more precarious and costly (Box 1) and sometimes unsuccessful. Individuals may succumb to MDR infections because all available drugs have failed, especially in the developing world ${ }^{1}$. Notable global examples include hospital and community MDR strains of Mycobacterium tuberculosis, Enterococcus faecium, Enterobacter cloacae, Klebsiella pneumoniae, S. aureus, Acinetobacter baumanii and Pseudomonas aeruginosa $a^{3,16-18}$ (Box 2, World Health Organization website). In developing countries, MDR enteric disease agents such as Salmonella enteritidis, Shigella flexneri and Vibrio cholerae threaten and circumvent public health measures.

Overall, in the United States and the United Kingdom, 40-60\% of nosocomial $S$. aureus strains are methicillin-resistant (MRSA) and usually $\mathrm{MDR}^{17,18}$. More deaths are associated with MRSA than with methicillin-sensitive strains ${ }^{19}$. A steadily increasing, small proportion of MRSA also now shows low-level resistance to vancomycin (the drug of choice), leading to treatment failure ${ }^{20,21}$. And in three US states, full-fledged vancomycin-resistant strains of $S$. aureus have appeared ${ }^{22,23}$, having acquired the resistance trait from vancomycinresistant enterococci. The latter, in particular MDR strains of E. faecium, have plagued clinicians treating immunocompromised individuals in hospitals in the United States and elsewhere for more than a decade ${ }^{24,25}$. At present, the newly developed drugs daptomycin, linezolid and the streptogramin combination, dalfopristin/quinopristin, can treat vancomycin-resistant enterococci, MRSA and vancomycin-resistant $S$. aureus, although some strains have emerged with resistance to the latter two agents ${ }^{26,27}$.

Among the Gram-negative bacteria, hospital infections caused by $P$. aeruginosa and A. baumanii are sometimes resistant to all, or all but one, antibiotics, which seriously challenges the treatment of immunocompromised individuals and can result in death ${ }^{3}$. The extendedspectrum $\beta$-lactamases, carried among Enterobacteriaceae such as 


\section{BOX 1 COSTS OF RESISTANCE}

Few studies have examined the costs of antibiotic resistance. One has noted that antibiotic-resistant infections double the duration of hospital stay, double mortality and probably double morbidity (and presumably the costs) as compared with drug-susceptible infections $^{88}$. A cost comparison of treating methicillin-resistant (MRSA) versus methicillin-susceptible (MSSA) S. aureus in New York City found almost a threefold increase in mortality $(21 \%$ versus $8 \%$ ) and an economic cost increase of $22 \%$ associated with MRSA. For all hospitalized individuals with MRSA in New York City, such costs would translate into millions of dollars 89 .

Another study has estimated the economic costs at US $\$ 150$ million to $\$ 30$ billion a year, depending on the number of deaths. It noted that the antimicrobial resistance selected in one year will persist, and subsequent years will bear the burden of the resistance problem of previous years ${ }^{90}$. The US Office of Technology Assessment advisory committee estimated a cost of several billion dollars when evaluating the effects of major resistant nosocomial agents ${ }^{48}$. If community infections are considered, the costs are even greater, particularly for combination therapies of multiple drugs such as those used to treat MDR tuberculosis and for treatments that substitute the parenteral route for the oral route.

Enterobacter and Klebsiella, destroy even the latest generations of penicillin and cephalosporins ${ }^{28-30}$. Of particular note is the increase in strains bearing metallo- $\beta$-lactamases that inactivate carbapenemsdrugs that are often the 'last resort' in serious infections of Gram-negative bacteria ${ }^{31,32}$.

The community has become similarly encumbered with MDR organisms. Some strains of E. coli, a common cause of urinary tract infection, resist members of six drug families including the more recently recommended fluoroquinolones. In parts of southeast Asia and China, $60-70 \%$ of E. coli are resistant to fluoroquinolones ${ }^{33}$; in the United States and other industrialized countries, frequencies approaching $10 \%$ are also worrisome because the trend jeopardizes the value of this drug family ${ }^{34,35}$.

Resistance in pneumococci continues to be an ever-increasing global threat that curtails treatment of pneumonias and ear infections, particularly in children. Having started with penicillin resistance, the organisms now tout resistance to macrolides and tetracyclines in many areas $^{36}$. One study has predicted that multidrug resistance-will override single-drug resistance in the present decade ${ }^{37}$. Strains of $N$. gonorrhoeae confront clinicians worldwide with triple resistance-to penicillins, tetracyclines and fluoroquinolones ${ }^{38,39}$. Because of the need to provide a single-dose therapy to this highly noncompliant population of infected individuals, a parenteral cephalosporin is the only treatment remaining. A recent report of decreased susceptibility to cefixime forewarns the future demise of this last-resort family of drugs for gonorrhea ${ }^{40}$.

Today, MRSA strains that differ from the hospital strains and possess a new virulence toxin (Panton-Valentine leukocidin) have emerged in communities of industrialized countries ${ }^{41,42}$. The socalled 'community-acquired MRSA' is resistant to the $\beta$-lactam antibiotics, requiring physicians to commence alternative therapies when MRSA is suspected. Children were found to succumb to communityacquired MRSA infection because the disease had become too far advanced by the time that another effective therapy was initiated ${ }^{43} . M$. tuberculosis, particularly in some endemic areas, bears resistance to as many as eight drugs, making some individuals with tuberculosis incurable ${ }^{14}$. Previously (inadequately) treated individuals are at greatest risk; in some areas, more than $50 \%$ of such individuals have MDR tuberculosis (Box 2).

The frequency of drug resistance in the community has extended the resistance problem beyond the confines of the hospital. Resistant strains can be traced from the community to the hospital and vice versa, indicating that drug resistance is no longer localized.

\section{What causes drug resistance?}

The resistance problem can be seen simplistically as an equation with two main components: the antibiotic or antimicrobial drug, which inhibits susceptible organisms and selects the resistant ones; and the genetic resistance determinant in microorganisms selected by the antimicrobial drug ${ }^{44,45}$. Drug resistance emerges only when the two components come together in an environment or host, which can lead to a clinical problem. Selected resistance genes and their hosts spread and propagate under continued antimicrobial selection to amplify and extend the problem to other hosts and other geographic locations. There are more than 15 classes of antibiotics ${ }^{1}$ whose targets are involved in essential physiological or metabolic functions of the bacterial cell (Table 1). None has escaped a resistance mechanism ${ }^{1}$. Millions of kilograms of antimicrobials are used each year in the prophylaxis and treatment of people, animals and agriculture globally $1,46-48$, driving the resistance problem by killing susceptible strains and selecting those that are resistant.

But how do bacteria acquire resistance? Drug resistance is mobile-the genes for resistance traits can be transferred among bacteria of different taxonomic and ecological groups by means of mobile genetic elements such as bacteriophages, plasmids, naked DNA or transposons ${ }^{1,49}$ (Box 3 ). These genes are generally directed against a single family or type of antibiotic, although multiple genes, each bearing a single drug resistance trait, can accumulate in the same organism. And, like the antibiotics themselves, resistance mechanisms are varied (Box 4).

In the absence of plasmids and transposons (which generally mediate high-level resistance), a step-wise progression from low-level to high-level resistance occurs in bacteria through sequential mutations in chromosomes ${ }^{1,33,50}$. This process was responsible for the initial emergence of penicillin and tetracycline resistance in N. gonorrhoeae. The organism later acquired transposons bearing genes with high-level resistance to these drugs. Strains of E. coli and other Enterobacteriaceae have evolved increasing resistance to fluoroquinolones, the result of mutations in the target enzymes (topoisomerases) and an increase in the expression of membrane proteins that pump the drugs out of the cell $^{33,50,51}$.

\section{BOX 2 DRUG RESISTANCE WEBSITES}

- Alliance for the Prudent Use of Antibiotics (APUA): http://www.apua.org

- World Health Organization, Antituberculosis drug resistance in the world. Third Global Report:

http://www.who.int/gtb/publications/drugresistance/2004/ drs_report_1.pdf

- Global Alliance of Antibiotic Resistance Data (GAARD): http://www.apua.org/Miscellaneous/GaardDesc.pdf

- Reservoirs of Antibiotic Resistance (ROAR): http://www.apua.org/ROAR/roarhome.htm 


\section{REVIEW}

Table 1 Major antibiotic families and their mechanisms of action

\begin{tabular}{ll} 
Mechanism of action & Antibiotic families \\
\hline Inhibition of cell wall synthesis & $\begin{array}{l}\text { Penicillins; cephalosporins; carbapenems; } \\
\text { daptomycin; monobactams; glycopeptides }\end{array}$ \\
\hline Inhibition of protein synthesis & $\begin{array}{l}\text { Tetracyclines; aminoglycosides; } \\
\text { oxazolidonones; streptogramins; ketolides; } \\
\text { macrolides; lincosamides }\end{array}$ \\
\hline Inhibition of DNA synthesis & Fluoroquinolones \\
\hline Competitive inhibition of folic & Sulfonamides; trimethoprim \\
acid synthesis & Rifampin \\
\hline Othibition of RNA synthesis & Metronidazole \\
\hline
\end{tabular}

Chromosomal mutants of $S$. aureus bearing intermediate resistance to vancomycin first appeared in response to vancomycin use $\mathrm{e}^{20}$, only to be followed by those that acquired the high-level resistance transposon from enterococci ${ }^{22,23}$. A small increase in the minimum inhibitory concentration to an antimicrobial should alert clinical microbiologists in hospitals and communities to an incipient problem of resistance. Although still classified as 'susceptible,' a strain with decreased susceptibility to a drug heralds the eventual emergence of higher-level resistance and should galvanize efforts towards altering the use of that antimicrobial in that environment.

Resistant bacteria accumulate multiple resistance determinants The long-term use of a single antibiotic (that is, for more than 10 days) will select for bacteria that are resistant not only to that antibiotic, but to several others ${ }^{1,52}$. This phenomenon was found to occur after the prolonged use of tetracycline for urinary tract infections $s^{53}$ and for acne $^{54}$. Under continued antimicrobial selection, the susceptible intestinal and/or skin flora may become colonized by organisms that are resistant not only to the ingested drug, but also to other, structurally unrelated drugs. In animals, MDR emerged after the application of subtherapeutic (growth promotion) levels of tetracyclines in feed ${ }^{55}$. Within days, chickens began excreting tetracycline-resistant E. coli; by two weeks, the excreted E. coli were resistant to several antibiotics.

This phenomenon reflects the linkage of different resistance genes on the same transposon or plasmid. It is unclear, however, why multiple resistance plasmids eventually emerge with the prolonged use of a single antimicrobial. Bacteria that are already resistant to one growthinhibitory agent seem to be favored in recruiting additional resistance traits from other bacteria sharing the environment: it was from the doubly resistant (penicillin and tetracycline) strains of N. gonorrhoeae that the new fluoroquinolone-resistant strains emerged.

\section{Loss of resistance is slow}

Resistant bacteria may rapidly appear in the host or environment after antibiotic use, but they are slow to be lost, even in the absence of the selecting antibiotic. This phenomenon reflects the minimal survival cost to the emerging resistant strains. In addition, as discussed above, resistance genes are often linked with genes specifying resistance to other antimicrobials or toxic substances on the same plasmids ${ }^{56}$. The presence of MDR plasmids assures maintenance of the plasmid as long as any one of the resistances provides a survival advantage to the host bacterium. This principle also applies to determinants of resistance to

\section{BOX 3 THE GENETICS AND SPREAD OF DRUG RESISTANCE}

Drug resistance genes can be spread from one bacterium to another through various mechanisms such as plasmids, bacteriophages, naked DNA or transposons. Some transposons contain integrons-more complex transposons that contain a site for integrating different antibiotic resistance genes and other gene cassettes in tandem for expression from a single promoter ${ }^{91}$. Originally discovered among Gram-negative bacteria, integrons have been since located in Gram-positive commensal flora-a newly found reservoir of these unique genetic elements ${ }^{92}$.

A model of resistance gene spread is the tet( $\mathrm{M})$ tetracycline resistance gene, which is commonly located on the transposon Tn916 (ref. 93). It has been found in Gram-positive and Gramnegative bacteria, aerobic and anaerobic bacteria, and in all environmental and biological niches ${ }^{94}$.

Chromosomal genes can be also transferred: they are acquired by one bacterium through the uptake of naked DNA released from another microorganism. This transfer process, called transformation, created penicillin-resistant S. pneumoniae through the acquisition of genes from the naturally occurring, penicillin-resistant commensal Streptococcus viridans and the formation of mosaic, penicillin-insensitive, penicillin-binding proteins ${ }^{95,96}$. Similar exchanges have helped to generate quinolone-resistant strains of $S$. pneumoniae through the production of a mutant drug-resistant gyrase ${ }^{97}$. Other organisms that are easily capable of integrating naked chromosomal DNA are $H$. influenzae and $A$. baumanii.

The pneumococci have shown that the chromosomal location of the resistance determinant is not a safeguard against its spread. Bacteria themselves are mobile and can easily travel from person to person and between countries. Resistant pneumococci in Iceland and in the United States have been shown to be the progeny of strains that initially appeared in Spain ${ }^{85}$. Thus, countries and citizens worldwide have become part of a global microbial ecology, sharing and spreading the consequences of antimicrobial resistance.

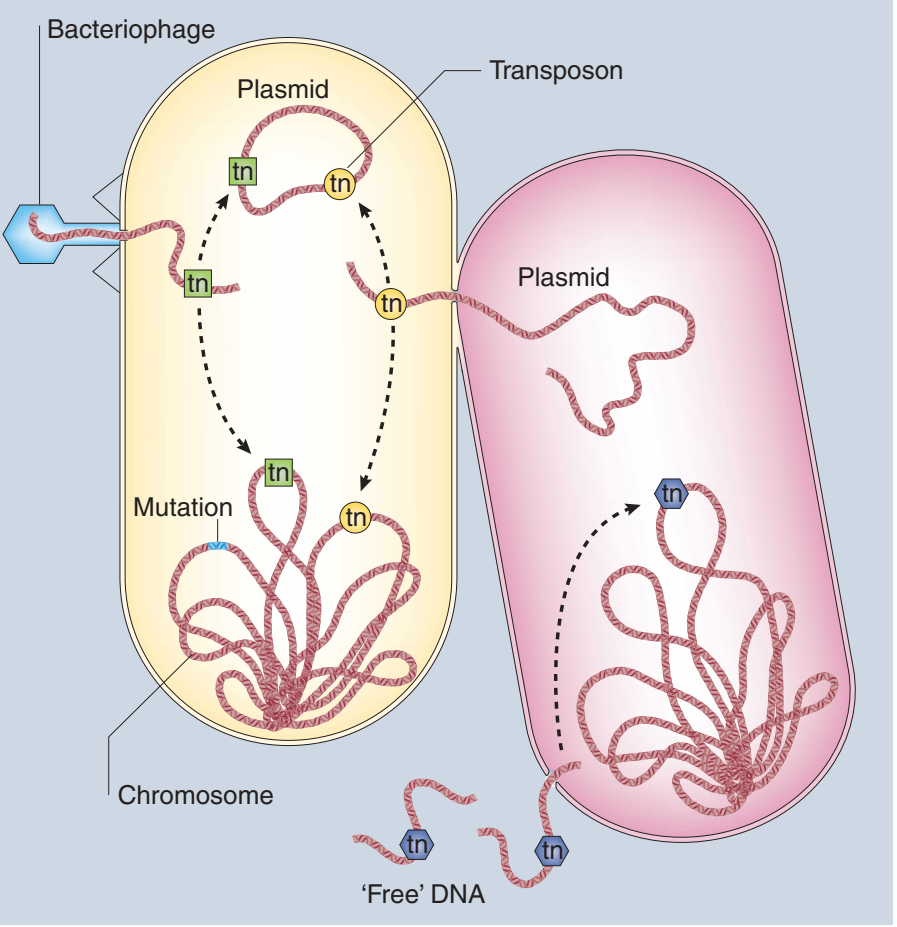




\section{BOX 4 BIOLOGICAL MECHANISMS OF RESISTANCE}

Resistance mechanisms vary. Some are directed at the antibiotic itself: enzymes such as $\beta$-lactamases destroy penicillins and cephalosporins, and modifying enzymes inactivate chloramphenicol and aminoglycosides such as streptomycin and gentamicin. Others target how the drug is transported; for example, an active efflux of drug mediates resistance to the tetracyclines, chloramphenicol and the fluoroquinolones ${ }^{98,99}$. A third type of mechanism (not shown) alters the intracellular target of the drug-for example, the ribosome, metabolic enzymes or proteins involved in DNA replication or cell wall synthesis-making the drug unable to inhibit a vital function in the microbial cell.

The same kind of drug resistance mechanism can be specified by many different genes. For example, the $\beta$-lactamases now number in the hundreds and more than 20 different resistance determinants mediate an efflux of tetracyclines ${ }^{100}$. In addition, more than one type of mechanism may provide resistance to the same antibiotic; for example, tetracycline resistance can be effected by either efflux or ribosome protection ${ }^{101}$. Although most fluoroquinolone resistance stems from chromosomal mutations in the gyrase target or from drug efflux, a plasmid-mediated resistance to fluoroquinolones has been recently described ${ }^{102}$. Since being recognized, it has been found among clinical strains of E. coli and K. pneumoniae ${ }^{103}$. Multidrug resistance can be specified by chromosomal genes for regulatory proteins such as MarA and SoxS. These proteins promote drug resistance by controlling the expression of other chromosomal genes, such as those involved in drug efflux ${ }^{61}$.

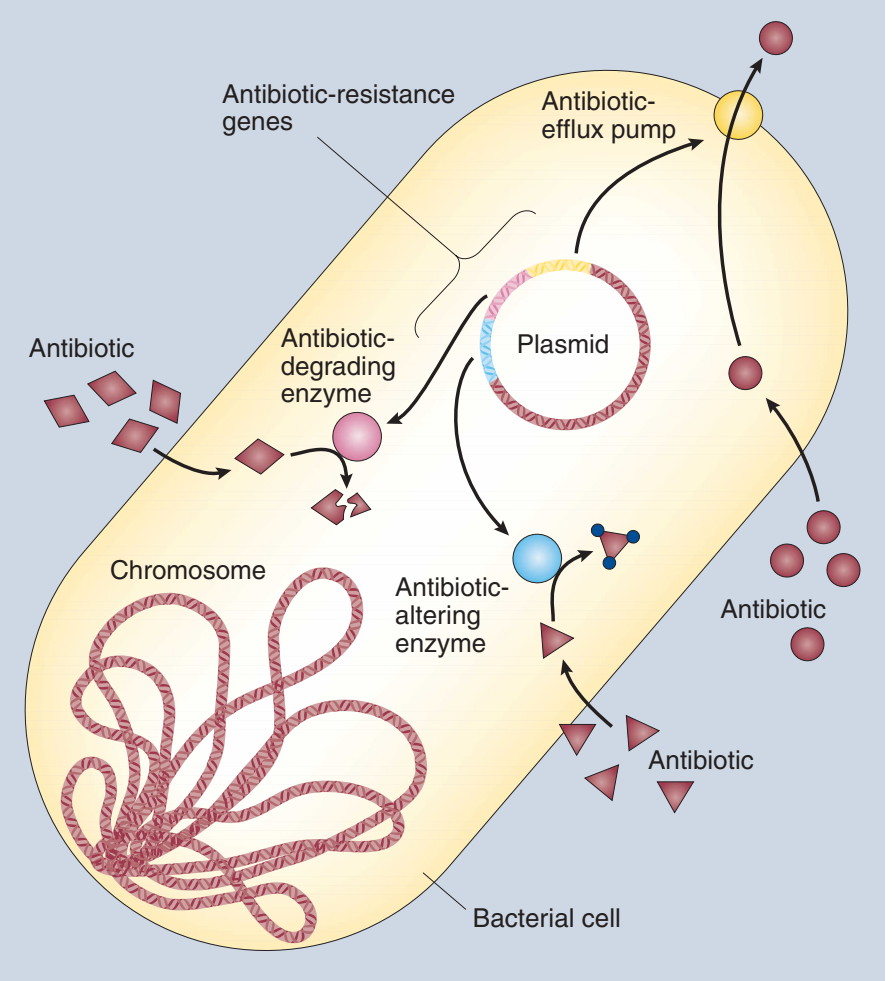

biocides such as quaternary ammonium compounds, because biocide efflux genes can be found on plasmids bearing genes for resistance to antibiotics in $S$. aureus $^{57}$.

Some studies have, however, tracked a decline in resistance frequencies when an antibiotic is removed ${ }^{58}$. A significant countrywide reversal of macrolide resistance in $S$. pyogenes resulted from a Finnish nationwide campaign to reduce macrolide usage. In 2 years, resistance declined from about $20 \%$ to less than $10 \%{ }^{59}$. Nonetheless, resistance generally persists at some low level and reintroduction of the antimicrobial will reselect resistant strains despite months or even years of nonuse.

Replacement by susceptible flora represents a chief contribution to a decrease in resistant strains. For example, despite being put into clean cages, chickens previously fed tetracycline-laced feed were found to continue to excrete tetracycline-resistant E. coli at high frequencies. When placed in separate cages and moved to a new location in the barn every 2-3 days, however, the resistance frequency dropped ${ }^{60}$. This 'dilution' of resistant strains was similarly accomplished by housing the chickens with greater numbers of cage mates that excreted susceptible flora. The findings suggest that the fastest way to eliminate resistant strains is to outnumber them with susceptible strains.

\section{The ecology of antibiotic resistance}

The impact of the drug selection process can be largely confined to the individual taking the antibiotic if widespread antibiotic usage is absent. After therapy, the selected resistant commensal strains will eventually be 'diluted out' and their growth will be suppressed by the return of drug-susceptible, natural competitors. If, however, whole populations are being treated with the same class of antibiotic, susceptible strains will have little opportunity to recolonize their niche and resistant strains will acquire an important advantage. The resulting ecological imbalance produces a potentially serious environmental pool of resistance genes ${ }^{61}$.

Ecologically speaking, it is the density of antibiotic usage that enhances resistance selection and its effects. The 'selection density' involves the total amount of antibiotic being applied to a geographically defined number of individuals in a setting, whether it is the home, daycare center, hospital or farm ${ }^{62}$ (Fig. 1). Each individual becomes a 'factory' of resistant bacteria that enter the environment. The disparity between resistance rates in the local community and those in city hospitals reflects differential ecological effects of antibiotic use. The end result of the selective pressure will reflect the number of individuals who are contributing resistant bacteria to that environment and the residual number of surviving, susceptible bacteria.

The ecological effects of antibiotics make them unique therapeutic agents. They are 'societal drugs' in which individual use affects others sharing that environment ${ }^{62,63}$. For example, antibiotic treatment for acne was found to produce an MDR skin flora not only in the individual with acne, but also in other members of the household ${ }^{64}$. High numbers of MDR bacteria were found in the intestinal flora of ambulatory individuals in the Boston area, even though none had recently taken an antibiotic ${ }^{65}$. In Nepal, resistance rates in individuals were found to correlate more with the total community use of antibiotics than with the individual's own use ${ }^{66}$.

In addition, the selection of resistance continues because antimicrobials persist, largely intact, in natural environments. Antimicrobials in waste waters are being reported with increasing frequency and are potentially important contributors to the environmental selection of antibiotic-resistant organisms ${ }^{67}$. The findings suggest that one approach to the antibiotic resistance problem could be to design drugs that self-destruct after treatment, thereby removing a contributing factor in the propagation of resistance. 


\section{REVIEW}

\section{Use of antibiotics in food animals and agriculture}

Considerable debate surrounds the relationship between antimicrobial use in animals and the resistance problem in people ${ }^{47}$. The chronic use of subtherapeutic amounts of antibiotics for growth promotion in food animals has been banned in the European Union, but it continues in the United States, albeit under intense scrutiny by the Center for Veterinary Medicine of the Food and Drug Administration. Despite their low-level application, the antibiotics select determinants mediating high-level, clinically relevant resistance $^{55}$. Enteric organisms such as Salmonella, Campylobacter, Listeria, enterococci and some strains of E. coli are propagated primarily among animals and subsequently infect people. The transfer may occur through the food chain or through animal handlers ${ }^{68-71}$. If the organisms are MDR, the emergence of their resistance results principally from use and overuse of antibiotics in the animals. Overall, animal contributions to the resistance problem in human infections are small but not insignificant; they have a major role if enteric organisms are involved.

Antibiotics also enter the environment through the dusting of fruit trees for disease prophylaxis ${ }^{72}$ and the application of antibiotic-laden animal manure on croplands ${ }^{1,47}$. These varied applications all add to the continued selection of resistant bacteria.

\section{How can we manage and prevent drug resistance?}

Track the resistance frequency. Local, national and global surveillance systems of drug susceptibility would help to communicate the current status of resistance in a location, facilitating more appropriate choices of treatment. Such surveillance would alert public health officials to new pathogens and would spur the implementation of control policies. In this regard, the Alliance for the Prudent Use of Antibiotics has established its Global Advisory on Antibiotic Resistance Data project to synthesize, evaluate and report the surveillance data from five large global surveillance systems (Box 2).

Commensal organisms are common reservoirs of antibiotic resistance plasmids, transposons and genes. E. coli and the enterococci of the gut serve as reservoirs from which several antibiotic resistance genes can spread ${ }^{73}$. The commensal Haemophilus parainfluenzae has been shown to confer $\beta$-lactamase-specifying plasmids to $H$. influenzae ${ }^{52}$. Similarly, Staphylococcus epidermidis serves as a reservoir for resistance genes and plasmids for the more pathogenic S. aureus ${ }^{52,74}$. Vancomycin resistance determinants found initially among enterococci appeared in other commensal bacteria before emerging in $S$. aureus ${ }^{9}$. This concept has been recently formalized by an Alliance for the Prudent Use of Antibiotics-based Reservoirs of Antibiotic Resistance project that supports studies examining the link between resistance in commensal flora and resistance in clinical isolates (Box 2).

Isolate hospitalized individuals with potentially dangerous resistant bacteria: cohorting. In Perth, Australia, hospital patients colonized with MRSA are isolated in special units, a process that has led to the lowest levels of MRSA and MDR staphylococci among all Australian hospitals ${ }^{75,76}$. Similar measures in Scandinavian countries and Holland protect hospitals from the entry and spread of resistant, difficult-to-treat infectious disease agents. In the United States, individuals with MRSA or vancomycin-resistant entercocci are housed in single rooms and kept microbiologically isolated. Although more than this single measure is needed to reduce the spread of MRSA, a review of this practice has identified six well-designed studies with a positive outcome and has concluded that cohorting should be "continued until further research establishes otherwise"77.
Introduce new therapeutic approaches. Confronted with a shortage of new antimicrobials, we must use our current drugs more prudently. Reducing and improving use can diminish resistance and permit a drug to resurface eventually as an effective therapy ${ }^{58}$. The appropriate use of the antibiotics not only can help to reverse high resistance frequencies, but also can curb the appearance of resistance to newer agents ${ }^{58}$. Decreasing antibiotic usage in the intensive care and other hospital units has shown that susceptible indigenous strains will repopulate the ecological niche in the absence of drug-selective pressure. But the process is slow and more difficult when addressing MDR strains, for which the use of many antibiotics must change to affect the presence of that strain. In addition, such efforts cannot succeed alone ${ }^{78}$. They need to be complemented by other actions (see below). For tuberculosis, better compliance after 'directly observed therapy' has clearly proved to be effective in treating the disease and in preventing the emergence of resistance ${ }^{79}$. Continued use of the same drugs in areas where resistance is endemic should be halted. From what we have learned, shorter-course therapies with highly active antibiotics will also reduce the pressure on multidrug resistance.

The development of new antibiotics-either those that block or circumvent resistance mechanisms or those that attack new targets-is essential. Such antibiotics would evade current resistance mechanisms, which can thwart the success of new, but structurally similar drugs. A different approach focuses on preventing infection by inhibiting key gene products that are involved in the infection process itself ${ }^{80}$. Because the inhibition of these targets does not affect growth, selection for resistance should be considerably reduced. The pipeline for new
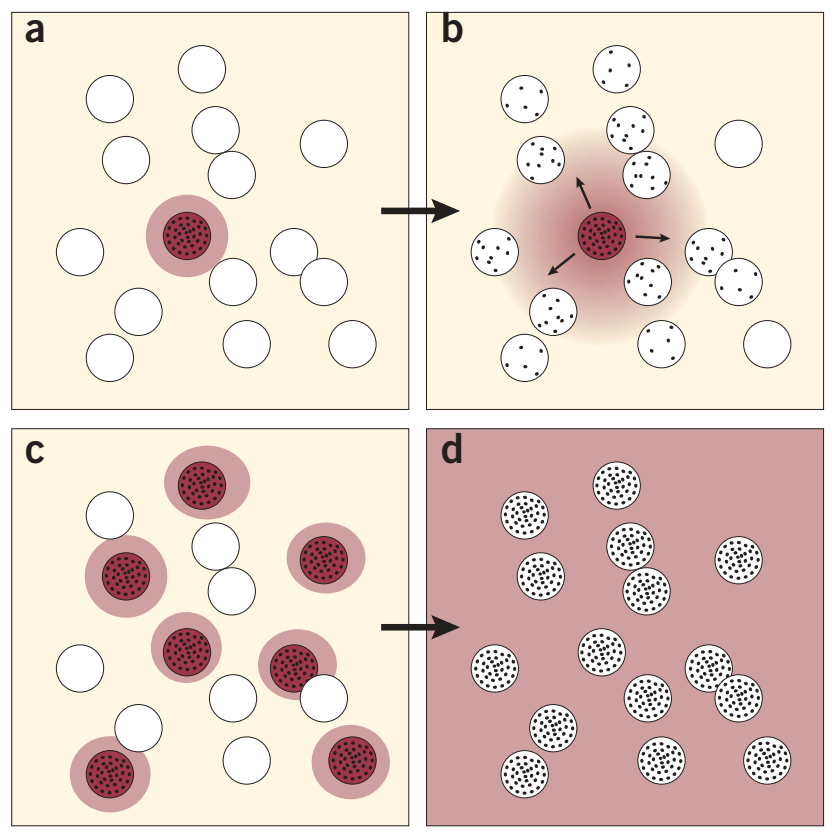

Figure 1 Post-therapeutic effects of antibiotic dispersion. (a) While on antimicrobial therapy, the individual (e.g. person or animal) is a focal point for a high concentration of both antibiotic (red shading) and resistant bacteria (black dots) that are selected and generated from its use. (b) Over time, resistant bacteria spread to local contacts and antibiotic enters the environment through waste and water disposal (for example, from animals) or sewage (from people). If several individuals are treated, however (c), a higher density of antimicrobial and resistant organisms is established in the same environment (d). The selective process is continuous, occurring both during and after therapy. 
drugs is small, because the major pharmaceutical companies have largely abandoned the antibiotic discovery field ${ }^{81}$. Fortunately, the need is being addressed by small, often start-up companies that can devote full attention to this goal but will ultimately require support from investors or from the larger pharmaceutical industry.

The availability of rapid diagnostics for the healthcare provider would greatly enhance the ability to prescribe more appropriately. A test to distinguish a viral from a bacterial infection, for example, one based on procalcitonin levels ${ }^{82}$, should decrease unnecessary antimicrobial use. More rapid susceptibility tests would aid the initial selection of an antibiotic. There is no better need for such diagnostics than for early stage tuberculosis, before the foci of resistant strains can spread out of control.

Finally, the development of conjugated vaccines, such as those based on encapsulated $H$. influenzae type $b$ and pneumococcus, can diminish bacterial disease and the consequent need for antibiotics. But vaccine development and delivery are problematic. In addition, with the transformation of commensal strains into pathogens in immunocompromised individuals, the activity of vaccines against these organisms could paradoxically destroy a natural defense against recognized pathogens.

\section{Perspectives}

The erosion of effective antimicrobials continues as we witness the increased frequency of resistance to all drugs-in particular, the fluoroquinolones, vancomycin and carbapenems, which are often the drugs of last resort. Imminent crises have been averted by new drugs that can combat MDR Gram-positive bacteria. With the relative absence of new antimicrobials coming to market and with new threats arising from the Gram-negative bacteria, however, the number of drug options leaves us perilously close to none or only a single effective agent for some life-threatening infections.

Hundreds of $\beta$-lactam-degrading enzymes are rapidly undermining the mainstay penicillins and late-generation cephalosporin agents. The increase in metallo- $\beta$-lactamases, which are active against carbapenems and most other $\beta$-lactams, is an alarming new development ${ }^{32}$. Colistin, a relatively toxic drug, has become a last-resort choice in treating some strains of $P$. aeruginos $a^{83}$. In addition, new types of highly virulent MRSA in the community are posing concerns for everyday activities among populations at risk, including children, contact sports participants, the military and economically deprived indigenous populations ${ }^{41,42}$. Notably, organisms that were formerly classified as primarily 'commensal', namely enterococci, pneumococci and E. coli, as well as environmental organisms such as P. aeruginosa and A. baumanii, have become emerging pathogens. The narrow focus on the older clinical pathogens must be broadened to accommodate the trend toward these newer disease agents, which are largely panresistant. From an ecological perspective, contamination of the environment with antibiotics from human, animal and agricultural spillover continues to exert selective pressure for resistance determinants.

Improved technologies have identified the clonal nature of infectious agents, enabling us to track their movement more closely and to understand better their epidemiology. Notable examples are MDR tuberculosis ${ }^{84}$, Streptococcus pneumoniae ${ }^{85}$ and some strains of cotrimoxazole-resistant E. coli ${ }^{86}$. Such advances have facilitated a broader view of resistance as an ecological problem. Few, if any, barriers are able to contain resistance genes and their bacterial hosts in our closely connected world.

Although drug resistance has been recognized since the early 1940s, and despite many national and international reports, including that of the World Health Organization ${ }^{87}$, urging ways to curtail it, the problem continues to grow and to evolve from one decade into the next.
The highly disease-oriented focus of modern medicine has hindered a clear perception of the enormity and all-encompassing nature of resistance, which suffers from an 'identity crisis.' Resistance is a nameless cloud that looms over otherwise controllable infections, but lacks the powerful status of a readily identifiable disease state to spur largescale efforts of control.

The role of antibiotics in the treatment of infectious diseases cannot be seen as anything but essential for the foreseeable future. The obstacles of few new antimicrobials on the horizon and the increasing frequency of multidrug resistance mean that we must redouble our efforts to preserve the agents at hand, while intensifying the search for new therapeutics.

\section{COMPETING INTERESTS STATEMENT}

The authors declare they have no competing financial interests.

Published online at http://www.nature.com/naturemedicine/

1. Levy, S. The Antibiotic Paradox: How Misuse of Antibiotics Destroys their Curative Powers (Perseus Cambridge, 2002).

2. Ash, C. (ed.) Trends in Microbiology vol.2, 341-422 (Elsevier, Cambridge, UK, 1994).

3. Levy, S.B. The challenge of antibiotic resistance. Sci. Am. 278, 46-53 (1998).

4. Levy, S.B. Microbial resistance to antibiotics. An evolving and persistent problem. Lancet 2, 83-88 (1982).

5. Barber, M. Infection by penicillin resistant Staphylococci. Lancet 2, 641-644 (1948).

6. Crofton, J. \& Mitchison, D.A. Streptomycin resistance in pulmonary tuberculosis. Br. Med. J. 2, 1009-1015 (1948).

7. Watanabe, T. Infective heredity of multidrug resistance in bacteria. Bacteriol. Rev. 27, 87-115 (1963).

8. Olarte, J. Antibiotic resistance in Mexico. APUA Newsletter 1, $3 \mathrm{ff}$ (1983).

9. Levy, S.B. Antibiotic resistance: consequences of inaction. Clin. Infect. Dis. $\mathbf{3 3}$ Suppl. 3, S124-S129 (2001).

10. Elwell, L., Roberts, M., Mayer, L. \& Falkow, S. Plasmid-mediated $\beta$-lactamase production in Neisseria gonorrhoeae. Antimicrob. Agents Chemother. 11, 528-533 (1977).

11. De Graaff, J., Elwell, L.P. \& Falkow, S. Molecular nature of two $\beta$-lactamase-specifying plasmids isolated from Haemophilus influenzae type b. J. Bacteriol. 126, 439-446 (1976).

12. Marshall, B., Roberts, M., Smith, A. \& Levy, S.B. Homogeneity of transferable tetracycline resistance determinants in Hemophilus species. J. Infect. Dis. 149, 1028-1029 (1984).

13. van Klingeren, B., van Embden, J.D. \& Dessens-Kroon, M. Plasmid-mediated chloramphenicol resistance in Haemophilus influenzae. Antimicrob. Agents Chemother. 11, 383-387 (1977).

14. Bloom, B.R. \& Murray, C.J.L. Tuberculosis: commentary on a re-emergent killer. Science 257, 1055-1064 (1992).

15. Iseman, M.D. Treatment of multidrug-resistant tuberculosis. N. Engl. J. Med. [erratum appears in N. Engl. J. Med. 329,1435 (1993)] 329, 784-791 (1993).

16. Walsh, F.M. \& Amyes, S.G.B. Microbiology and drug resistance mechanisms of fully resistant pathogens. Curr. Opin. Microbiol. 7, 439-444 (2004).

17. Weinstein, R.A. Controlling antimicrobial resistance in hospitals: infection control and use of antibiotics. Emerg. Infect. Dis. 7, 188-192 (2001).

18. Anonymous. European Antimicrobial Resistance Surveillance System. EARSS Annual Report 2002 (2002).

19. Cosgrove, S.E. et al. Comparison of mortality associated with methicillin-resistant and methicillin-susceptible Staphylococcus aureus bacteremia: a meta-analysis. Clin. Infect. Dis. 36, 53-59 (2003).

20. Hiramatsu, K. Vancomycin resistance in staphylococci. Drug Resist. Updat. 1, 135-150 (1998).

21. Fridkin, S.K. Vancomycin-intermediate and -resistant Staphylococcus aureus: what the infectious disease specialist needs to know. Clin. Infect. Dis. 32, 108-115 (2001).

22. Weigel, L.M. et al. Genetic analysis of a high-level vancomycin-resistant isolate of Staphylococcus aureus. Science 302, 1569-1571 (2003).

23. Tenover, F.C. et al. Vancomycin-resistant Staphylococcus aureus isolate from a patient in Pennsylvania. Antimicrob. Agents Chemother. 48, 275-280 (2004).

24. Arthur, M. \& Courvalin, P. Genetics and mechanisms of glycopeptide resistance in enterococci. Antimicrob. Agents Chemother. 37, 1563-1571 (1993).

25. Goossens, H. The epidemiology of vancomycin-resistant enterococci. Curr. Opin. Infect. Dis. 12, 537-541 (1999).

26. Jones, R.N., Ballow, C.H., Biedenbach, D.J., Deinhart, J.A. \& Schentag, J.J. Antimicrobial activity of quinupristin-dalfopristin (RP 59500, Synercid) tested against over 28,000 recent clinical isolates from 200 medical centers in the United States and Canada. Diagn. Microbiol. Infect. Dis. 31, 437-451 (1998). 
27. Meka, V.G. \& Gold, H.S. Antimicrobial resistance to linezolid. Clin. Infect. Dis. 39, 1010-1015 (2004)

28. Bush, K. New $\beta$-lactamases in Gram-negative bacteria: diversity and impact on the selection of antimicrobial therapy. Clin. Infect. Dis. 32, 1085-1089 (2001).

29. Paterson, D.L. et al. International prospective study of Klebsiella pneumoniae bacteremia: implications of extended-spectrum $\beta$-lactamase production in nosocomial Infections. Ann. Intern. Med. 140, 26-32 (2004).

30. Bradford, P.A. Extended-spectrum $\beta$-lactamases in the 21st century: characterization, epidemiology and detection of this important resistance threat. Clin. Microbiol. Rev. 14, 933-951 (2001).

31. Nordmann, P. \& Poirel, L. Emerging carbapenemases in Gram-negative aerobes. Clin. Microbiol. Infect. 8, 321-331 (2002).

32. Livermore, D.M. \& Woodford, N. Carbapenemases: a problem in waiting? Curr. Opin. Microbiol. 3, 489-495 (2000).

33. Wang, H., Dzink-Fox, J.L., Chen, M. \& Levy, S.B. Genetic characterization of highly fluoroquinolone-resistant clinical Escherichia coli strains from China: role of acrR mutations. Antimicrob. Agents Chemother. 45, 1515-1521 (2001).

34. Zervos, M.J. et al. Relationship between fluoroquinolone use and changes in susceptibility to fluoroquinolones of selected pathogens in 10 United States teaching hospitals, 1991-2000. Clin. Infect. Dis. 37, 1643-1648 (2003).

35. Karlowsky, J.A., Kelly, L.J., Thornsberry, C., Jones, M.E. \& Sahm, D.F. Trends in antimicrobial resistance among urinary tract infection isolates of Escherichia coli from female outpatients in the United States. Antimicrob. Agents Chemother. 46, 2540-2545 (2002).

36. Schrag, S.J. et al. Emergence of Streptococcus pneumoniae with very-high-level resistance to penicillin. Antimicrob. Agents Chemother. 48, 3016-3023 (2004).

37. McCormick, A.W. et al. Geographic diversity and temporal trends of antimicrobial resistance in Streptococcus pneumoniae in the United States. Nat. Med. 9, 424-430 (2003)

38. Tanaka, M., Nakayama, H., Haraoka, M. \& Saika, T. Antimicrobial resistance of Neisseria gonorrhoeae and high prevalence of ciprofloxacin-resistant isolates in Japan, 1993 to 1998. J. Clin. Microbiol. 38, 521-525 (2000).

39. Centers for Disease Control and Prevention. Increases in fluoroquinolone-resistant Neisseria gonorrhoeae-Hawaii and California. Morb. Mortal. Wkly. Rep. 51 , 1041-1044 (2002)

40. Wang, S.A. et al. Multidrug-resistant Neisseria gonorrhoeae with decreased susceptibility to cefixime-Hawaii, 2001. Clin. Infect. Dis. 37, 849-852 (2003).

41. Vandenesch, F. et al. Community-acquired methicillin-resistant Staphylococcus aureus carrying Panton-Valentine leukocidin genes: worldwide emergence. Emerg. Infect. Dis. 9, 978-984 (2003).

42. Herold, B. et al. Community-acquired methicillin-resistant Staphylococcus aureus in children with no identified predisposing risk. J. Am. Med. Assoc. 279, 593-598 (1998).

43. Anonymous. From the Centers for Disease Control and Prevention. Four pediatric deaths from community-acquired methicillin-resistant Staphylococcus aureusMinnesota and North Dakota, 1997-1999. J. Am. Med. Assoc. 282, 1123-1125 (1999).

44. Levy, S.B. Balancing the drug resistance equation. Trends Microbiol. 2, 341-342 (1994).

45. Levy, S.B. The 2000 Garrod lecture. Factors impacting on the problem of antibiotic resistance. J. Antimicrob. Chemother. 49, 25-30 (2002).

46. Mellon, M., Benbrook, C. \& Benbrook, K.L. Hogging it: estimates of antimicrobial abuse in livestock. (UCS Publications, Cambridge, UK, 2001).

47. Barza, M., S.L. Gorbach. The need to improve antimicrobial use in agriculture: ecological and human health consequences. Clin Infect. Dis. 34, S71-S144 (2002)

48. US Congress. Office of Technology Assessment. Impacts of Antibiotic Resistant Bacteria (OTA-H-629, US Government Printing Office, Washington, DC, 1995).

49. Levy, S.B. \& Miller, R.V. (eds.) Gene Transfer in the Environment (McGraw Hill, New York, 1989).

50. Schneiders, T., Amyes, S.G.B. \& Levy, S.B. Role of AcrR and RamA in fluoroquinolone resistance in clinical Klebsiella pneumoniae isolates from Singapore. Antimicrob. Agents Chemother. 47, 2831-2837 (2003).

51. Piddock, L.J. Mechanisms of fluoroquinolone resistance: an update 1994-1998. Drugs 2 Suppl. 2, 11-18 (1999).

52. Levy, S.B. Ecology of plasmids and unique DNA sequences. in Engineered Organisms in the Environment: Scientific Issues (eds. Halvorson, H.O., Pramer, D. \& Rogul, M.) 180-190 (ASM Press, Washington DC, 1985).

53. Datta, N. et al. R factors in Escherichia coli in faeces after oral chemotherapy in general practice. Lancet 1, 312-315 (1971).

54. Moller, J.K., Bak, A.L., Stenderup, A., Zachariae, H. \& Afzelius, H. Changing patterns of plasmid-mediated drug resistance during tetracycline therapy. Antimicrob. Agents Chemother. 11, 388-391 (1977).

55. Levy, S.B., FitzGerald, G.B. \& Macone, A.B. Changes in intestinal flora of farm personnel after introduction of a tetracycline-supplemented feed on a farm. N. Engl. J. Med. 295, 583-588 (1976)

56. Summers, A.O. Generally overlooked fundamentals of bacterial genetics and ecology. Clin. Infect. Dis. 34 Suppl 3, S85-S92 (2002).

57. Sidhu, M.S., Heir, E., Leegaard, T., Wiger, K. \& Holck, A. Frequency of disinfectant resistance genes and genetic linkage with $\beta$-lactamase transposon Tn552 among clinical staphylococci. Antimicrob. Agents Chemother. 46, 2797-2803 (2002).

58. Barbosa, T.M. \& Levy, S.B. The impact of antibiotic use on resistance development and persistence. Drug Resist. Updat. 3, 303-311 (2000).

59. Seppala, H. et al. The effect of changes in the consumption of macrolide antibiotics on erythromycin resistance in group A streptococci in Finland. Finnish Study
Group for Antimicrobial Resistance. N. Engl. J. Med. 337, 441-446 (1997).

60. Levy, S.B. Emergence of antibiotic-resistant bacteria in the intestinal flora of farm inhabitants. J. Infect. Dis. 137, 689-690 (1978).

61. Alekshun, M.N. \& Levy, S.B. Regulation of chromosomally mediated multiple antibiotic resistance: the mar regulon. Antimicrob. Agents Chemother. 41, 2067-2075 (1997).

62. Levy, S.B. Antibiotic resistance: an ecological imbalance, in Antibiotic Resistance: Origins, Evolution, and Spread, 1-9 (J. Wiley, Chichester, UK, 1997).

63. Levy, S.B. Starting life resistance-free. N. Engl. J. Med. 323, 335-337 (1990).

64. Miller, Y.W. et al. Sequential antibiotic therapy for acne promotes the carriage of resistant staphylococci on the skin of contacts. J. Antimicrob. Chemother. 38 , 829-837 (1996).

65. Levy, S.B., Marshall, B., Schluederberg, S., Rowse, D. \& Davis, J. High frequency of antimicrobial resistance in human fecal flora. Antimicrob. Agents Chemother. 32, 1801-1806 (1988).

66. Walson, J.L., Marshall, B., Pokhrel, B.M., Kafle, K.K. \& Levy, S.B. Carriage of antibiotic-resistant fecal bacteria in Nepal reflects proximity to Kathmandu. $J$. Infect. Dis. 184, 1163-1169 (2001).

67. Kummerer, K. \& Henninger, A. Promoting resistance by the emission of antibiotics from hospitals and households into effluent. Clin. Microbiol. Infect. 9, 1203-1214 (2003).

68. Rolland, R.M., Hausfater, G., Marshall, B. \& Levy, S.B. Antibiotic-resistant bacteria in wild primates: increased prevalence in baboons feeding on human refuse. Appl. Environ. Microbiol. 49, 791-794 (1985).

69. Corpet, D.E. Antibiotic resistance from food. N. Engl. J. Med. 318, 1206-1207 (1988)

70. Levy, S.B. Antibiotic resistant bacteria in food of man and animals, in Antimicrobials and Agriculture (ed. Woodbine, M.) 525-531 (Butterworths, London, 1984).

71. Marshall, B., Petrowski, D. \& Levy, S.B. Inter- and intraspecies spread of Escherichia coli in a farm environment in the absence of antibiotic usage. Proc. Natl. Acad. Sci. USA 87, 6609-6613 (1990).

72. Vidaver, A. Uses of antimicrobials in plant agriculture. Clin. Infect. Dis. 34, S107-S110 (2002).

73. DeFlaun, M.F. \& Levy, S.B. Genes and their varied hosts, in Gene Transfer in the Environment (eds. Levy, S.B. \& Miller, R.V.) 1-32 (McGraw-Hill, New York, 1989).

74. Cohen, M.L., Wong, E.S. \& Falkow, S. Common R-plasmids in Staphylococcus aureus and Staphylococcus epidermidis during a nosocomial Staphylococcus aureus outbreak. Antimicrob. Agents Chemother. 21, 210-215 (1982).

75. Pearman, J.W. \& Grubb, W.B. Preventing the importation and establishment of methicillin-resistant Staphylococcus aureus (MRSA) in hospitals in Western Australia. APUA Newsletter 11, 1-2 (1993).

76. Turnidge, J., Lawson, P., Munro, R. \& Benn, R. A national survey of antimicrobial resistance in Staphylococcus aureus in Australian teaching hospitals. Med. J. Aust. 150, 69-72 (1989).

77. Cooper, B.S. et al. Isolation measures in the hospital management of methicillin resistant Staphylococcus aureus (MRSA): systematic review of the literature. Br. Med. J. 329, 533 (2004).

78. Livermore, D. Can better prescribing turn the tide of resistance? Nat. Rev. Microbiol. 2, 73-78 (2004).

79. Initial therapy for tuberculosis in the era of multidrug resistance. Recommendations of the Advisory Council for the Elimination of Tuberculosis. MMWR Recomm. Rep. 42, 1-8 (1993).

80. Alekshun, M.S.B.L. Targeting virulence to prevent infection: to kill or not to kill? Drug Discovery Today: Therapeutic Strategies (in the press) (2004).

81. Projan, S.J. Why is big Pharma getting out of antibacterial drug discovery? Curr. Opin. Microbiol. 6, 427-430 (2003).

82. Simon, L., Gauvin, F., Amre, D.K. \& Saint-Lous, P. \& Lacroix, J. Serum procalcitonin and C-reactive protein levels as markers of bacterial infection: a systematic review and meta-analysis. Clin. Infect. Dis. 39, 206-217 (2004).

83. Markou, N. et al. Intravenous colistin in the treatment of sepsis from multiresistant Gram-negative bacilli in critically ill patients. Crit. Care 7, R78-R83 (2003).

84. Bifani, P.J. et al. Origin and interstate spread of a New York City multidrug-resistant Mycobacterium tuberculosis clone family. J. Am. Med. Assoc. 275, 452-457 (1996)

85. Soares, S., Kristinsson, K.G., Musser, J.M. \& Tomasz, A. Evidence for the introduction of a multiresistant clone of serotype 6B Streptococcus pneumoniae from Spain to Iceland in the late 1980s. J. Infect. Dis. 168, 158-163 (1993).

86. Manges, A.R. et al. Widespread distribution of urinary tract infections caused by a multidrug-resistant Escherichia coli clonal group. N. Engl. J. Med. 345, 1007-1013 (2001).

87. WHO. Global Strategy for Containment of Antimicrobial Resistance (WHO, Geneva, 2001).

88. Holmberg, S.D., Solomon, S.L. \& Blake, P.A. Health and economic impacts of antimicrobial resistance. Rev. Infect. Dis. 9, 1065-1078 (1987).

89. Rubin, R.J. et al. The economic impact of Staphylococcus aureus infection in New York City hospitals. Emerg. Infect. Dis. 5, 9-17 (1999).

90. Phelps, C.E. Bug/drug resistance. Sometimes less is more. Med. Care 27, 194-203 (1989).

91. Hall, R.M. et al. Mobile gene cassettes and integrons in evolution. Ann. NY Acad. Sci. 870, 68-80 (1999).

92. Nandi, S., Maurer, J.J., Hofacre, C. \& Summers, A.O. Gram-positive bacteria are a major reservoir of Class 1 antibiotic resistance integrons in poultry litter. Proc. Natl. Acad. Sci. USA 101, 7118-7122 (2004). 


\section{REVIEW}

93. Clewell, D.B. \& Gawron-Burke, C. Conjugative transposons and the dissemination of antibiotic resistance in streptococci. Annu. Rev. Microbiol. 40, 635-659 (1986).

94. Roberts, M.C. Tetracycline resistance determinants: mechanisms of action, regulation of expression, genetic mobility, and distribution. FEMS Microbiol. Rev. 19, 1-24 (1996).

95. Dowson, C.G., Coffey, T.J. \& Spratt, B.G. Origin and molecular epidemiology of penicillin-binding-protein-mediated resistance to $\beta$-lactam antibiotics. Trends Microbiol. 2, 361-366 (1994).

96. Spratt, B.G. Resistance to antibiotics mediated by target alterations. Science $\mathbf{2 6 4}$ 388-393 (1994)

97. Balsalobre, L., Ferrandiz, M.J., Linares, J., Tubau, F. \& de la Campa, A.G. Viridans group streptococci are donors in horizontal transfer of topoisomerase IV genes to Streptococcus pneumoniae. Antimicrob. Agents Chemother. 47, 2072-2081 (2003).
98. Levy, S.B. Active efflux mechanisms for antimicrobial resistance. Antimicrob. Agents Chemother. 36, 695-703 (1992).

99. Nikaido, H. Multidrug efflux pumps of gram-negative bacteria. J. Bacteriol. 178, 5853-5859 (1996).

100. Levy, S.B. et al. Nomenclature for new tetracycline resistance determinants. Antimicrob. Agents Chemother. 43, 1523-1524 (1999).

101. McMurry, L.M. \& Levy, S.B. Tetracycline resistance in gram-positive bacteria, in Gram-Positive Pathogens (ASM Press, Washington DC, 2000).

102. Martinez-Martinez, L., Pascual, A. \& Jacoby, G.A. Quinolone resistance from a transferable plasmid. Lancet 351, 797-799 (1998).

103. Wang, M., Sahm, D.F., Jacoby, G.A. \& Hooper, D.C. Emerging plasmid-mediated quinolone resistance associated with the qnr gene in Klebsiella pneumoniae clinical isolates in the United States. Antimicrob. Agents Chemother. 48, 1295-1299 (2004). 